\title{
INQUÉRITO SOBRE O CONSUMO DE ALIMENTOS POSSÍVEIS DE CONTAMINAÇÃO POR MICOTOXINAS NA INGESTA ALIMENTAR DE ESCOLARES DA CIDADE DE LAVRAS, MG ${ }^{1}$
}

\author{
Inquiry on the consumption of meals susceptible to contamination by mycotoxins in alimentary \\ ingesta of scholars of the city of Lavras, MG
}

\author{
Rozane Aparecida da Silva², Sara Maria Chalfoun ${ }^{3}$, Marcelo Augusto Mendes da Silva ${ }^{4}$, \\ Marcelo Cláudio Pereira ${ }^{5}$
}

\begin{abstract}
RESUMO
Com presente trabalho, objetivou-se investigar a alimentação de alunos em idade escolar, de quatro escolas da rede estadual e particular da cidade de Lavras, $M G$, supostamente contaminada com micotoxinas $\mathrm{O}$ universo amostral constou de escolares de $1^{\mathrm{a}}$ e $2^{\mathrm{a}}$ séries do ensino fundamental, totalizando 197 alunos. A avaliação do consumo alimentar dos escolares foi efetuada utilizando-se dois tipos de inquéritos dietéticos: o recordatório de 24 horas e o questionário de frequiência alimentar (QFA). De posse dos dados referentes do recordatório de 24 horas e o QFA, promoveu-se uma seleção entre os alimentos citados, caracterizando-os como alimentos com maior potencial de risco com relação à contaminação com micotoxinas. As análises descritivas da população de escolares indicaram uma população com boa distribuição amostral em função do sexo com 45,2\% (meninos) e 54,8\% (meninas). Não foi observada uma relação entre o grau de escolaridade das mães e o padrão alimentar dos escolares, uma vez que 60,9\% das mães apresentaram um nível de escolaridade correspondente ao $1^{\circ}$ grau do ensino fundamental. Dos alimentos selecionados no grupo de risco de contaminação de micotoxinas, observou-se que 49,7\% das crianças entrevistadas apresentaram uma ingestão superior a 72,6\% do seu valor calórico total (VCT) obtido de alimentos desse grupo embora tenham sido observadas dissimilaridades entre as escolas com relação aos alimentos ingeridos. As análises do consumo energético individual apresentaram uma ingestão de alimentos de risco acima de 72,5\%, sendo 41,5\% representados na Escola A, 61\% na Escola B, 33,3\% na Escola C e 64,7\% na Escola D. Conclui-se, nesta pesquisa, que a população apresenta um perfil de alta ingestão de alimentos considerados de risco para micotoxinas, existindo a necessidade de adequação da dieta, visando reduzir a exposição a esse risco.
\end{abstract}

Termos para indexação: Micotoxinas, consumo alimentar, escolares.

\begin{abstract}
The present work was intended to investigate the feeding of students at school age from four state and private network schools of the town of Lavras, MG, supposedly contaminated with mycotoxins. The sample universe comprised elementary school 1 st and $2^{\text {nd }}$ grade students amounting to 197 students. The evaluation of the students' consumption was performed by utilizing two sorts of dietary inquires: the 24-hour recordatory and the feeding frequency questionnaire (FFQ). In possession of the data concerning the 24-hour recordatory and the FFQ, promoted a selection among the quoted foods, characterizing them as foods with greater potential of risk as regards the contamination with mycotoxins. The descriptive analyses of the population of students at school indicated a population with a good sample distribution as related with sex with $45.2 \%$ ( boys) and $54.8 \%$ (girls). No relationship between degree of education of the mothers and the feeding standard of the students correspondent to the $1^{\text {st }}$ degree of elementary teaching. Out of the feeds selected in the group of mycotoxin contamination risk, it was found that $49.7 \%$ of the children interviewed presented a intake superior to $72.6 \%$ of its total caloric value (TCV) obtained from feeds of that group although they have been observed dissimilarities among the schools with relation to the feeds ingested. The analyses of the individual energy consumption presented a risk intake of foods above $72.5 \%, 41.5 \%$ being stood for at School A, $61 \%$ at School B, 33.3\% at School C and $64.7 \%$ at School D. It follows, in this research, that the population presents a profile of high intake of foods considered of risk for mycotoxins, existing the need of adequacy of the diet, aiming to reduce the exposition to that risk.
\end{abstract}

Index terms: Mycotoxins, feeding, students at school.

(Recebido em 22 de setembro de 2004 e aprovado em 10 de agosto de 2005)

\section{INTRODUÇÃO}

A ocorrência de algumas espécies de fungos em alimentos tem servido de alerta para o risco de contaminação com micotoxinas, contribuindo para a perda de qualidade do produto e prejuízos à saúde dos seres humanos. Os fungos são capazes de produzir um amplo conjunto de substâncias biologicamente ativas. Estes metabólitos biológicos, dos quais as micotoxinas representam a maior parte, possuem efeitos antipredatórios e pró-territoriais, garantindo a perpertuação do fungo.

\footnotetext{
${ }^{1}$ Parte da Tese de Doutorado do primeiro autor apresentado à Universidade Federal de Lavras/UFLA - Cx. P. 3037 - $37200-000$ - Lavras, MG. ${ }^{2}$ Bióloga - Departamento de Ciência dos Alimentos/DCA - Universidade Federal de Lavras/UFLA - Cx. P. 3037 - $37200-000$ - Lavras, MG rozane@ufla.br

${ }^{3}$ Pesquisadora da Empresa de Pesquisa Agropecuária de Minas Gerais / FAPEMIG - chalfoun@ufla.br

${ }^{4}$ Professor, Nutricionista do Centro Universitário de Lavras/UNILAVRAS - Rua Padre José Poggel, 506 - Centenário - $37200-000$ - Lavras, MG.

${ }^{5}$ Pesquisador da Empresa de Pesquisa Agropecuária de Minas Gerais/EPAMIG.
} 
Os diferentes fungos produtores de micotoxinas são encontrados em todas as regiões do mundo e podem crescer em uma grande variedade de substratos e sob várias condições de umidade, $\mathrm{pH}$ e temperatura. Assim, os alimentos estão sujeitos à invasão por fungos e contaminação com micotoxinas no campo, durante e após a colheita, no processamento, no transporte e na estocagem, quando não são empregadas técnicas adequadas de manuseio.

As investigações sobre incidência de micotoxinas em alimentos e rações são de suma importância para que esforços possam ser concentrados na prevenção, no controle da contaminação ou na destoxificação dos produtos susceptíveis a esse tipo de contaminação. Neste sentido, e com vistas a garantir a confiabilidade dos resultados, relativos à incidência de micotoxinas em alimentos, estudos visando aprimorar a metodologia para sua detecção e quantificação são, sem dúvida, urgentes e necessários.

Atualmente, cerca de 300 micotoxinas já foram isoladas, contudo, as toxinas mais conhecidas encontradas em alimentos e que comprovadamente têm propriedades tóxicas acentuadas e que estão mais largamente distribuídas nos alimentos causando danos ao consumidor são as toxinas do Ergot, aflatoxinas, esterigmatocistina, ocratoxina, zearalenona, tricotecenos, fumonisinas, patulina, toxinas produzidas no arroz, rubratoxinas, esporodesminas, ácido ciclopiazônico e micotoxinas tremorgênicas (SCUSSEL et al., 1983).

As aflatoxinas são metabólitos secundários sintetizados por fungos, principalmente do gênero Aspergillus flavus e A. parasiticus. Tais toxinas são responsáveis por graves intoxicações e têm se mostrado carcinogênicas, mutagênicas e teratogênicas a diversas espécies animais, inclusive ao homem.

A contaminação de produtos, como milho, amendoim, castanha-do-brasil e outros, por aflatoxinas, vem dificultando a exportação dos mesmos a países desenvolvidos, onde há rígido controle dos limites de tolerância de aflatoxinas.

A presença de aflatoxina no leite é de extrema relevância, pelo fato de lactação e alimentação serem dois processos concomitantes. Além disso, o leite é alimento básico para crianças e recém-nascidos que, por analogia com animais domésticos, devem ser mais susceptíveis que os adultos aos efeitos tóxicos das aflatoxinas. (PARREIRAS, 1985).

Dados epidemiológicos têm demonstrado correlação considerável entre contaminação de aflatoxinas nas rações e incidência de carcinoma hepático em várias regiões do mundo (BULLERMAN, 1974; BUTLER \& NEAL, 1977; SHANK, 1998; WOGAN et al., 1971).

As aflatoxinas provocam hepatoxicoses agudas e são conhecidas por ser carcinogênicas em algumas espécies de animais, tais como ratos e cobaias. Estes compostos são severamente tóxicos para os seres humanos, e são, provavelmente, responsáveis por necrose no fígado, seguida por uma exposição crônica, e pode estar envolvida na epidemiologia de câncer no fígado de humanos em algumas partes do mundo, talvez em sinergismo com o vírus da hepatite B (MOSS, 1996).

Uma vez ingerida na dieta, a aflatoxina $\mathrm{B} 1$ pode ser convertida em um epóxido, possivelmente carcinogênico e conhecido por reagir com um resíduo da guanina no DNA.

A Patulina, outro metabólito carcinogênico é produzida por diferentes espécies de Penicillium, Aspergilllus e Byssochlamys são capazes de produzir patulina, dentre elas o Penicillium expansum que é comum na maçã deteriorada (TANIWAKI et al., 1989). A patulina apresenta ação antibiótica contra diferentes bactérias e fungos, mas por outro lado, demonstra alta toxicidade para plantas, tecidos e células de animais (ENGEL \& TEUBER, 1984).

A patulina tem sido encontrada em sucos e purês de maçã em níveis que variam de < 1 a 3.993 mg/L (BURDA, 1992).

No Brasil, Machinsky \& Midio (1992) analisaram 22 amostras de suco de maçã, das quais duas apresentaram teores de 8 e $10 \mathrm{mg}$ de patulina por litro de suco.

O ácido ciclopiazônico é uma substância produzida por várias espécies de Penicillium e de Aspergillus (TRUCKSESS et al., 1987).

Estudos com espécies aflatoxigênicas e não aflatoxigênicas de Aspergillus flavus têm mostrado que existem cepas que podem produzir apenas aflatoxinas, apenas CPA, as duas toxinas ou nenhuma delas (GALLACHER et al., 1978).

Rao \& Husain (1985) associaram a síndrome "kodua poisoning" em animais e seres humanos com a presença de ácido ciclopiazônico presente em sementes de "Kodo millet" (Paspalum scobriculatum) em certas regiões da Índia (teor de ácido ciclopiazônico não especificado). Aspergillus flavus e A. tamarii, ambos produtores de ácido ciclopiazônico, também foram isolados das sementes tóxicas. Esta toxina já foi detectada em vários alimentos, como queijos, carnes, amendoim e milho (URANO et al., 1992).

Outra micotoxina com propriedades tóxicas acentuadas, largamente distribuída na natureza e nos alimentos, é a ocratoxina A. 
As ocratoxinas foram descobertas na África do Sul, em 1965, por um grupo de cientistas que freqüentemente isolavam cepa de Aspergillus ochraceus, responsáveis por certos efeitos tóxicos em animais de laboratório, subseqüentemente, identificando o metabólito (SCUSSEL, 1998).

Tem sido relatado de maneira crescente que a OTA, devido ao seu efeito hepatotóxico, nefrotóxico, teratogênico e carcinogênico, é considerada um fator de risco para a saúde humana (XIAO et al., 1996).

A ocratoxina pode ser encontrada principalmente em milho, cevada, feijão, amendoim, café, soja, trigo sarraceno, centeio, arroz, sorgo, castanha do Pará, superfície de presunto, pimentões vermelhos, pimenta do reino, pimenta preta, suco de maçã, suco de uva, aveia, pão, semente de papoula, nozes, cerveja, vegetação em decomposição, solo e ervilha. Os níveis comumente encontrados são abaixo de $27,5 \mathrm{mg} / \mathrm{kg}$.

A OTA tornou-se um problema de saúde pública mundial desde que foi associada com nefropatologia dos Balcans (KROGH et al., 1997). Vários trabalhos têm sido desenvolvidos para documentar a ocorrência de OTA em alimentos para o homem e em rações para animais.

A toxicidade da ocratoxina A pode ter três grandes efeitos: a) inibição na síntese de proteínas, b) inibição na síntese de ATP e c) peroxidação lipídica (XIAO et al., 1996).

A fumonisina é outro metabólito secundário do gênero Fusarium. Nijs et al. (1996), revisando trabalhos de vários autores, relataram que 25 espécies isoladas em produtos e subprodutos naturais de milho biossintetizam micotoxinas em condições controladas de laboratório. Entre estas espécies, em ordem decrescente de frequiência de citação bibliográfica, o $F$. verticillioides tem habilidade de produzir zearalenona, toxina T-2 e fumonisinas B1, B2, B3, B4; C1 e o F. Proliferatum têm habilidade de produzir fumonisinas B1, B2 e B3.

A fumonisina B1 causa leucoencefalomalácia em equinos, síndrome do edema pulmonar em suínos, câncer no fígado em ratos e está estatisticamente correlacionada com o aumento do risco de câncer do esôfago em seres humanos que consumiram milho contaminado em Transkei, na África do Sul (MARASAS, 1995).

Segundo Marasas (1995), a International Agency for Research on Câncer (IARC) classificou a fumonisina no grupo $2 \mathrm{~B}$, considerando-a como possível ação carcinogênica em seres humanos.

Apesar de existirem relativamente poucas publicações mostrando o impacto de micotoxinas em alimentos no Brasil, é relevante citar que estudos sobre micotoxinas vêm crescendo de ano para ano. Encontros e simpósios são organizados, indústrias se preocupam em obedecer às normas com receio de perderem sua fatia no mercado, cientistas estão buscando novas formas de amenizar o problema, desenvolvendo novas tecnologias e cursos estão sendo criados. Desta forma, a presente pesquisa teve como objetivo propor um levantamento da ingesta de alimentos, em algumas escolas da cidade de Lavras,MG, que são mais propensos a contaminação com micotoxinas.

\section{MATERIAIS E MÉTODOS}

$\mathrm{O}$ universo amostral constou de escolares de $1^{\circ}$ e $2^{\circ}$ séries do ensino fundamental, totalizando 197 alunos.

A avaliação do consumo alimentar dos escolares foi efetuada utilizando-se a média de dois tipos de inquéritos dietéticos: o recordatório de 24 horas (GIBSON, 1990; KRAUSE \& MAHAN, 2002) e o questionário de frequiência alimentar (QFA) (GIBSON, 1990).

O registro de consumo de alimentos em domicílio, dos escolares das quatro escolas, constou de uma entrevista com a mãe ou pessoa responsável pela alimentação. Segundo Krall et al. (1988) e Madden et al. (1976), o registro é um instrumento de apoio utilizado para as limitações da memória das crianças principalmente das mais jovens.

O QFA constou de 84 alimentos, incluindo sazonalidade e hábitos alimentares regionais, sobre os quais foram coletadas informações referentes à frequiência de consumo e ao tamanho das porções consumidas.

De posse dos dados referentes ao recordatório de 24 horas e o QFA, promoveu-se uma seleção entre os alimentos citados, daqueles cujos registros anteriores permitiram caracterizá-los como alimentos com maior potencial de riscos com relação à contaminação com micotoxinas.

\section{RESULTADOS E DISCUSSÃO}

Inicialmente serão apresentadas as análises descritivas da população dos escolares. Após, serão apresentadas as análises referentes ao consumo de alimentos de risco.

É interessante notar que há maior percentual de mulheres em 3 escolas, sendo 56,6\% na escola A, 53,7\% na escola $\mathrm{B}$ e 57,8 na escola $\mathrm{C}$, as quais constituem a maioria dos participantes. Dados do IBGE (FUNDAÇÃO IBGE, 2000) mostram que dos 14.481 alunos matriculados no ensino fundamental a maior representatividade é feminina.

Segundo Tellartolli Junior et al. (1996), a escolaridade é um importante indicador do nível sócio- 
econômico de uma população, pois está relacionada à renda, acesso a informações e utilização dos serviços de saúde.

O nível de escolaridade da mãe na determinação do estado nutricional das crianças é de fundamental importância, uma vez que o grau de educação da família e sua condição sócio-econômica têm efeitos consideráveis sobre o modo de vida e hábitos alimentares das crianças. (MONTEIRO et al., 2000).

A proporção de mães com baixa escolaridade é uma realidade na população em estudo; 60,9\% delas apresentaram um nível de escolaridade correspondente ao ensino fundamental.

Pelos resultados de avaliação do consumo alimentar dos escolares (recordatório de 24 horas e QFA), observou-se um consumo em freqüências variáveis de alimentos, dos quais foram selecionados aqueles considerados como de maior risco quanto ao potencial de contaminação por micotoxinas.

Tendo como base as informações obtidas nos mesmos registros por meio dos formulários utilizados anteriormente, elaborou-se uma tabela de porcentagem da presença de determinados alimentos de risco para micotoxinas, na freqüência de consumo (diário, semanal, quinzenal, mensal e nunca/raramente), apresentados na Tabela 1.

Dos alimentos listados, verifica-se que o arroz, feijão, manteiga e pão francês foram os mais freqüentes na alimentação da família e, conseqüentemente das crianças; o consumo diário deles foi mencionado por mais de $90 \%$ dos entrevistados. O leite e o café também tiveram um consumo bastante expressivo; mais de $75 \%$ dos entrevistados fazem o uso diário desses alimentos.

Por outro lado, alimentos como o amendoim, que tradicionalmente é conhecido como de alto risco de contaminação por aflatoxinas, apresentou uma frequiência bastante modesta. Apenas $0,5 \%$ da população faz o consumo diário e mais de $90 \%$ consome este alimento mensalmente ou não consome. A ocorrência da menor freqüência do consumo de amendoim pode estar associada à época do ano em que a pesquisa foi realizada.

TABELA 1 - Freqüência das médias do consumo alimentar dos alimentos considerados de risco para micotoxinas através de inquéritos alimentares. Lavras,MG, 2004.

\begin{tabular}{|c|c|c|c|c|c|}
\hline \multirow{2}{*}{ Alimentos } & \multicolumn{5}{|c|}{ Freqüiência de consumo (\%) } \\
\hline & Diária & Semanal & Quinzenal & Mensal & Nunca/Rara \\
\hline Amendoim & 0,5 & 1 & 3,5 & 37,5 & 57,4 \\
\hline Arroz & 98,5 & 1,5 & - & - & - \\
\hline Café & 76,1 & 16,2 & 4,1 & 1,0 & 2,5 \\
\hline Carne de boi & 10,7 & 26,4 & 32,0 & 13,7 & 17,3 \\
\hline Castanha-do-pará & 2,0 & 3,0 & 1,0 & 2,0 & 91,5 \\
\hline Cevada & 0,5 & 0,5 & 0,5 & 1,0 & 97,5 \\
\hline Farinha de mesa & 9,1 & 25,9 & 36,0 & 11,2 & 17,8 \\
\hline Feijão & 96,9 & 3,0 & - & - & - \\
\hline Frango & 13,2 & 49,2 & 24,9 & 10,2 & 2,5 \\
\hline Hambúrguer & 1,5 & 10,1 & 17,8 & 57,4 & 13,2 \\
\hline Iogurte & 20,8 & 47,5 & 19,3 & 7,1 & 5,1 \\
\hline Leite & 86,8 & 10,7 & 0,5 & 0,5 & 1,5 \\
\hline Maçã & 38,6 & 27,9 & 18,3 & 6,6 & 8,6 \\
\hline Manteiga & 95,4 & 2,5 & - & 1,0 & 1,0 \\
\hline Milho & 5,1 & 18,8 & 29,4 & 18,8 & 27,9 \\
\hline Ovos & 23,9 & 67,5 & 6,1 & 1,0 & 1,5 \\
\hline Pão francês & 95,4 & 2,0 & 1,0 & 0,5 & 1,0 \\
\hline Pão doce & 45,2 & 12,7 & 16,8 & 5,1 & 20,3 \\
\hline Pipoca & 4,6 & 20,8 & 37,1 & 25,4 & 12,2 \\
\hline Pizza & - & 9,1 & 18,8 & 54,3 & 17,8 \\
\hline Polenta & 20,3 & 52,8 & 14,7 & 4,6 & 7,6 \\
\hline Queijo & 34,0 & 31,6 & 16,8 & 11,2 & 6,6 \\
\hline
\end{tabular}

Obs.: Os traços (-) significam que não foi observado o alimento na refeição. 
Entre as micotoxinas relacionadas com alimentos, as quais apresentam probabilidade de ter grande significado para a saúde humana nos países tropicais encontra-se, a ocratoxina A (OTA), toxina de ocorrência natural, podendo crescer em cereais e seus produtos, leguminosas e outros alimentos, inclusive no café.

É interessante mencionar que, na maioria das famílias amostradas, observou-se um consumo diário expressivo de café, com freqüência de $76,1 \%$. É pertinente destacar o consumo de café na escola A $(81,1 \%)$, na escola B $(63,4 \%)$, na escola C $(84,4 \%)$ e na escola D $(100 \%)$.

Avaliando a presença de ocratoxina A em café solúvel normal e café solúvel descafeinado, Freitas (2000) afirmou que o valor médio de contaminação representa $8 \%$ da ingestão diária provisória estabelecida pelo Codex Alimentarius para a ocratoxina, levando em consideração o consumo de 5 xícaras de café por um consumidor adulto de $70 \mathrm{~kg}$.

Considerando-se a elevada freqüência de consumo de café detectada e que outros alimentos que participam da dieta são fontes potenciais desta micotoxina, especial atenção deve ser dispensada quanto à qualidade do produto adquirido, uma vez que esta bebida estará na dieta como uma provável fonte adicional de ocratoxina A. Os alimentos como amendoim e seus subprodutos, o milho, farelo de algodão, nozes, sementes oleaginosas, fenos, sorgo e feijão, estão incluídos nos principais relatos de ocorrência de aflatoxina, sempre que ocorram condições favoráveis de umidade e temperatura.

O consumo da polenta também surgiu uma freqüência bastante significativa, pois em $20 \%$ da população tem o hábito de ingerir alimentos à base de milho diariamente e 52,8\%, semanalmente. Confirma-se assim, uma generalização de seu consumo.

Com relação ao feijão, os percentuais de consumo diário verificados para cada escola foram Escola A, 96,2\%; Escola B, 96,3\%; Escola C, 97,8\% e Escola D, 100. É importante ressaltar que o feijão é a fonte mais importante de ferro da dieta da população brasileira.

Segundo um alerta da Organização Mundial de Saúde (FAO/OMS), pessoas que consomem alimento contendo $1.7 \mathrm{ppm}$ de aflatoxina (mesmo que por um curto período de tempo) poderão vir a sofrer de sérios problemas no fígado. Uma única dose de 75 ppm pode até causar morte (SANTURIO et al., 1999).

Com relação ao milho, nota-se que o consumo quinzenal é bastante expressivo quando observado nas Escolas A (37,7\%), Escola C (37,8\%), Escola D (58,8\%); somente na Escola B o consumo mensal observado foi de
26,8. Deve-se ressaltar que a farinha de milho apresenta um consumo bastante elevado, quando observado o consumo quinzenal nas quatro escolas.

Estes resultados conduzem a uma preocupação quanto à presença em freqüência relativamente alta pelos escolares deste cereal, somados ao fato de os grãos serem o principal substrato para a síntese de aflatoxinas e conseqüente elevação de risco de ocorrência de patologias humanas (MOREIRA et al., 2003; SANTURIO 1999).

De acordo com os resultados obtidos, verifica-se que, entre os produtos lácteos, o leite aparece com um consumo diário de 84,9\% (Escola A), (Escola B) 89,0\%, (Escola C) $80 \%$ e (Escola D) $100 \%$. Alimentos como o iogurte e o queijo são também bastantes freqüentes no consumo diário dos escolares.

De forma semelhante às considerações feitas para a presença de milho e derivados em freqüência relativamente elevada, observaram-se freqüências igualmente elevadas para o leite e derivados. Embora seja um alimento altamente nutritivo, pode ser o agente causador de diversos processos fisiológicos nas pessoas que os consomem (PARREIRAS, 1985). A contaminação do leite é resultado do consumo de alimentos (rações) contaminadas pelos animais e, segundo Martins \& Martins (1986), o teor de aflatoxina M1 excretada no leite é diretamente proporcional a quantidade de aflatoxina B1 ingerida.

Verificou-se que, nos consumos das escolas A, B, C e D o pão francês aparece com um percentual superior a 90\% nas refeições. Pereira (2001), estudando aflatoxina no pão, constatou que as aflatoxinas migram para o substrato adjacente e não ficam retidas no micélio dos fungos. A velocidade de migração na massa crua do pão, no pão pronto e nas nozes do coco é de vários centímetros em poucos dias. Por exemplo, foram achados $45.000 \mathrm{mg}$ de aflatoxina B1/kg no pão integral a 7 centímetros do micélio após uma migração de 9 dias. Por esta razão, não basta rejeitar a camada superficial contaminada por fungos (SCUSSEL, 1998).

Com relação ao consumo de pizzas, constata-se um consumo mensal observado na Escola A foi de 39,6\%, na Escola B de 73,2\%, na Escola C de $37,8 \%$ e na e Escola D de $52,9 \%$. Deve-se ressaltar que os produtos de panificação possuem duas fontes de contaminação que são a matériaprima utilizada para a sua confecção (farinhas e outros ingredientes) e a recontaminação durante o período de resfriamento nas panificadoras (PEREIRA, 2001), exigindo um controle de ambas.

A maçã é uma excelente fonte nutricional e tem interesse econômico, no entanto, Shank et al. (1998) 
ressaltam um risco freqüente de produção de patulina em maçãs nas temperaturas de refrigeração. Portanto, há necessidade de melhor controle, a fim de evitar constantes ingestões de toxina, uma vez que o consumo mensal no presente trabalho constatou-se elevado.

Entre as castanhas diversas, a castanha-do-pará é o produto com maior incidência de aflatoxinas no Brasil (CALDAS et al., 2002). Contudo, ela apresentou um baixo nível de ingestão no universo pesquisado, limitando assim a importância direta desse tipo de contaminação para a saúde dos escolares em estudo.

O consumo de ovos apresentou uma ingestão diária de 70,6\% na Escola D, ingestão semanal de 50,9\% na Escola A, $81,7 \%$ na Escola B e 75,6\% na Escola C. Sabe-se que a transferência das micotoxinas para os ovos é menos constante que no leite. (IDENTIFICANDO..., 2002) relataram que a quantidade de aflatoxina nos ovos aumentavam linearmente conforme a concentração de aflatoxina aumentava na ração. Estudos demonstraram que a maioria da aflatoxinas residem na clara rica em proteína. A Desoxinivalenol (DON), quando administrada em poedeiras, pode ser detectada após 24 horas; ela se prende na gema em forma de glicoranídeo conjugado, subestimando a quantidade de micotoxinas transferidas de ração para o alimento.

Com relação ao consumo de carnes bovinas e de hambúrguer, observou-se, uma ingesta elevada de carne de boi, com 32\% de ingesta semanal na Escola A, 39,0\% de ingesta quinzenal na Escola B, 42,2\% da ingesta semanal na Escola C e 76,5\% da ingesta diária na Escola D. Para o hambúrguer, observou-se uma alta ingestão mensal para as escolas A,B e C e somente a escola D com $70,6 \%$ da ingestão quinzenal.

A grande preocupação com o consumo da carne bovina é que depois do consumo e da absorção de micotoxinas pelos animais, a biotransformação dessas micotoxinas aumenta a toxicidade e tornando-as mais prontamente depositadas nos tecidos usados como alimento humano, produzido por animais que ingerem rações contaminadas. O mesmo ocorre com as aves, (IDENTIFICANDO..., 2002) resultando em um problema grave que é a transferência de micotoxinas para a cadeia alimentar humana, por meio da carne e latícinios.

A análise apresentada na Tabela 2, é importante para bipolarizar a exposição ao risco de alto consumo de micotoxinas. A partir desta análise, atribuiu-se uma mediana do percentual de calorias de risco ingeridas em $72,6 \%$ (as variáveis kcalind e kcalexp foram definidas em função do ponto de corte na mediana de kcalind). Assim, $50 \%$ da população ingere menos que $72,6 \%$ das calorias totais como calorias de risco de micotoxinas. A outra metade da população ingere mais que isso. Este resultado demonstra que a população, como um todo, tem uma tendência a ingerir alimentos com risco de presença de micotoxinas.

Por meio do recordatório 24 horas observou-se que 49,7\% das crianças entrevistadas apresentaram uma ingestão superior a 72,6\% do seu valor calórico total (VCT) de alimentos com risco de estarem contaminados com micotoxinas. Esses valores podem ser observados na Tabela 2 .

$\mathrm{Na}$ análise do consumo energético individual, com ingestão de alimentos de risco acima de 72,5\% (Tabela 3), 41,5\% foram observadas na Escola A, 61\% de ingestão na Escola B, 33,3\% na Escola C e 64,7\% na Escola D. A prevalência de consumo de dietas compostas com 76,2\% de alimentos supostamente de risco com micotoxinas evidenciou uma alta ingestão desses alimentos classificados de alto risco.

O predomínio de alimentos associados na faixa de risco de micotoxinas presentes na dieta das famílias dos escolares poderia ser potencializado considerando-se a correlação entre aflatoxinas e desnutrição crônica, uma vez que a ingesta acima do limite de 72,6\% ocorreu em quase $50 \%$ do universo amostral.

TABELA 2 - Percentual médio de kcal proveniente dos alimentos de risco em relação à quantidade total de calorias ingeridas. Lavras, MG, 2004.

\begin{tabular}{lcc}
\hline Ingestão de alimentos de risco com micotoxinas (kcal) & $(\%)$ & $\mathbf{N}$ \\
\hline 1 Acima de $72,6 \%$ de kcal & 49,7 & 98 \\
2 Abaixo de $72,6 \%$ de kcal & 50,3 & 99 \\
\hline Total & 100 & 197 \\
\hline
\end{tabular}

Ciênc. agrotec., Lavras, v. 31, n. 2, p. 439-447, mar./abr., 2007 
TABELA 3 - Percentual médio de kcal proveniente dos alimentos de risco em relação à quantidade de calorias ingeridas, por escola. Lavras,MG, 2004.

\begin{tabular}{|c|c|c|c|c|c|c|c|c|}
\hline \multirow[t]{3}{*}{$\begin{array}{c}\text { Ingestão de alimentos de risco } \\
\text { com micotoxinas } \\
\end{array}$} & \multicolumn{8}{|c|}{ Escolas } \\
\hline & \multicolumn{2}{|c|}{$\mathbf{A}$} & \multicolumn{2}{|c|}{$\mathbf{B}$} & \multicolumn{2}{|c|}{$\mathbf{C}$} & \multicolumn{2}{|c|}{ D } \\
\hline & $\%$ & $\mathbf{N}$ & $\%$ & $\mathbf{N}$ & $\%$ & $\mathbf{N}$ & $\%$ & $\mathbf{N}$ \\
\hline 1 Acima de $72,6 \%$ & 41,5 & 22 & 61,0 & 50 & 33,3 & 15 & 64,7 & 11,0 \\
\hline 2 Abaixo de $72,6 \%$ & 58,5 & 31 & 39,0 & 32 & 66,7 & 30 & 35,3 & 6,0 \\
\hline Total & 100 & 53 & 100 & 82 & 100 & 45 & 100 & 17 \\
\hline
\end{tabular}

\section{CONCLUSÕES}

A população apresenta uma bipolarização do perfil de ingestão de alimentos de risco, em que existe uma alta ingestão de alimentos considerados de risco para micotoxinas. Como $50 \%$ da população ingere menos que $72,6 \%$ dos calorias de risco e a outra metade ingere mais do que isso, então pode-se concluir que existe uma grande tendência à ingestão de alimentos de risco. Portanto, existe uma grande preocupação quando estima-se que cerca de $35 \%$ dos casos de câncer humano estejam diretamente relacionados à dieta, e a presença de principalmente aflatoxinas em alimentos é considerada um fator importante na incidência de câncer hepático.

A presença de micotoxinas, como a aflatoxina nos alimentos destinados ao consumo humano, supõe um risco óbvio do potencial para a saúde pública. Porém, desconhece-se até que ponto se manifesta esse potencial. No entanto só o fato de existirem constitui uma forte e suficiente motivação para se utilizar todos meios possíveis para preveni-las.

A presente pesquisa não nos possibilitou conhecer até que ponto os alimentos ingeridos potencializam o risco para a saúde pública. $\mathrm{O}$ simples fato de existir constitui uma forte e suficiente motivação para futuras pesquisas no sentido de explorar mais este universo promissor

As escolas A e D apresentaram maior ingestão de alimentos de risco que as escolas B e C.Todas as escolas diferiram quanto aos tipos de alimentos ingeridos.

O grau de escolaridade das mães não interferiu no padrão de consumo alimentar, não contribuindo para a ingestão de alimentos de risco.

\section{REFERÊNCIAS BIBLIOGRÁFICAS}

BULLERMAN, L. B. Inhibition of aflatoxin production by cinnamon. Journal of Food Science, Chicago, v. 39, n. 6, p. 1163-1165, Nov./Dec. 1974.
BURDA, K. Incidence of patulin in apple, pear, and mixed fruit products marketd in new South Wales. Journal of Food Protection, Ames, v. 55, n. 10, p. 796-798, Oct. 1992.

BUTLER, W. H.; NEAL, G. E. Modem of action an human health aspects of aflatoxin carcinogenesis. Pure and Applied Chemistry, Oxford, v. 49, n. 11, p. 1747-1751, 1977.

CALDAS, E. D.; SILVA, S. C.; OLIVEIRA, J. N. Aflatoxins and ochratoxin $\mathrm{A}$ in food and the risks to human health. Revista de Saúde Pública, São Paulo, v. 36, n. 3, p. 319-323, June 2002.

ENGEL, G.; TEUBER, M. Patulin and other small lactones. In: BETINA, V. Mycotoxins: production, isolation, separation and purification. Amsterdam: Elsevier Science, 1984. cap. 13, p. 291-314.

FUNDAÇÃO IBGE. Crianças e adolescentes: indicadores sociais. Belo Horizonte, 2000.

FREITAS, R. F. Fungos associados a grãos de café (Coffea arábica $\mathrm{L}$.) beneficiado de diversos municípios da região Sul de Minas Gerais. 2000. 72 p. Dissertação (Mestrado em Ciência dos Alimentos) - Universidade Federal de Lavras, Lavras, 2000.

GALLAGHER, R. T.; RICHARD, J. L.; STAHR, H. M.; COLE, R. J. Cyclopiazonic acid production by aflatoxigenic and non-aflatoxigenic strains of Aspergillus flavus. Mycopathologia, Dordrecht, v. 66, n. 1/2, p. 31-36, 1978.

GIBSON, R. S. Principles of nutritional assessment. New York: Oxford University, 1990.

IDENTIFICANDO um adsorvente melhor de micotoxinas. Mycotoxin Monthly, [S.1.], v. 5, n. 1, [2002]. Paper. 
KRALL, E. A.; DWYERT, J. T.; COLEMAN, K. A. Factors influencings accuracy of dietary recall. Nutrition Research, Oxford, v. 8, p. 829-841, 1988.

KRAUSE, M. V.; MAHAN, L. K. Alimentos, nutrição \& dietoterapia. 10. ed. São Paulo: Roca, 2002.

KROGH, P.; HALD, B.; PLESTINA, R.; CEOVIC, S. Balkan (endemic) nephropathy and foodborne ochratoxin A: preliminary results of a survey of foodstuffs. Acta Pathologica et Microbiologica Scandinavica, Section B: Microbiology, Copenhagen, v. 85, p. 238-240, 1997.

MACHINSKY, M.; MIDIO, A. F. Determinação de patulina em suco de maçã. In: ENCONTRO NACIONAL DE MICOTOXINAS, 7., 1992, São Paulo. Resumos... São Paulo: [s.n.], 1992.

MADDEN, J. P.; GOODMAN, S. J.; GUTHRIE, H. A. Validity of the 24-hs. Recall: analysis of data obtained from elderly subjects. Journal of the American Dietetics Association, Chicago, v. 68, n. 2, p. 143-147, 1976.

MARASAS, W. F. O. Fumonisins: their implications for human and animal health. Natural Toxins, New York, v. 3, n. 4, p. 193-198, July/Aug. 1995.

MARTINS, J. L. S.; MARTINS, I. S. Aflatoxina M1 no leite tipo "B" comercializado no município de São Paulo-SP. Revista Saúde Pública, São Paulo, v. 20, n. 4, p. 303-308, 1986.

MONTEIRO, C. A.; MONDINI, L.; COSTA, R. D. L. Mudanças na composição e adequação nutricional da dieta familiar nas áreas metropolitanas do Brasil: 1988-19996. Revista de Saúde Pública, São Paulo, v. 34, n. 3, p. 252-258, 2000.

MOREIRA, A. N.; RODRIGUES, K. L.; KAEFER, R. I.; MARQUES, A. P.; TEIXEIRA, L. S.; RODRIGUES, M. J.; CARVALHAL, J. B.; ALEIXO, J. A. G. Aflatoxinas, contaminação fúngica e teor de umidade em produtos utilizados como fonte de fibra alimentar. Revista Higiene Alimentar, São Paulo, v. 17, n. 104/105, p. 94-96, jan./fev. 2003.

MOSS, M. O. Mycotoxins. Mycological Research, Cambridge, v. 100, n. 5, p. 513-523, May 1996.
NIJS, M. de; ROMBOUTS, F.; NOTERMANS, S. Fusarium molds and their mycotoxins. Journal of Food Safety, Trimbull, v. 16, n. 1, p. 15-58, May 1996.

PARREIRAS, J. F. M. Ocorrência de aflatoxinas M1 e B1 em leite e forragens na micro-região de Viçosa-MG. Viçosa: UFV, 1985.

PEREIRA, M. C. Efeito da adição de condimentos no controle de microrganismos, na conservação de produtos de panificação e na inibição de metabólitos produzidos por fungos associados ao café. 2001. Dissertação (Mestrado) - Universidade Federal de Lavras, Lavras, 2001.

RAO, B. L.; HUSAIN, A. Presence of cyclopiazonic acid in kodo millet (Paspaluem scrobiculatum) causing "Kodua poisoning" in man and its production by associated fungi. Mycopathologia, Dordrecht, v. 89, p. 177-180, 1985.

SANTURIO, J. M.; MALLMAN, C. A.; ROSA, A.; APPEL, G. H.; DAGEFORDE, S.; BOOTTCHER, M. Effect of sodium bentonite on the performance and blood parameters of broiler chickens intoxicted with aflatoxins. British Poultry Science, London, v. 40, p. 115-119, 1999.

SCUSSEL, V. M.; RODRIGUEZ-AMAIA, D. B.; SILVA, W. J. da. Incidência de aflatoxinas em milho (Zea mays L.) e em seus produtos derivados, comercializados na região de Campinas, estado de São Paulo, Brasil. Ciência e Tecnologia de Alimentos, Campinas, v. 6, n. 1, p. 75-85, 1983.

SCUSSEL, V. M. Micotoxinas em alimentos. Florianópolis: Insular, 1998.

SHANK, R. C. et al. Dietary aflatoxins and human liver cancer: I. toxigenic moulds in foods and foods tuffs of tropical Southeast Asia. Food and Cosmetics Toxicology, Elmsford, v. 10, p. 51-60, 1998.

TANIWAKI, M. H.; BLEINROTH, E. W.; MARTIN, Z. J. Bolores produtores de patulina em maçã e suco industrialisado. Coletânea do Instituto de Tecnologia dos Alimentos, Campinas, v. 9, n. 1, p. 42-49, jan./jun. 1989.

TELAROLLI JÚNIOR, R.; MACHADO, C. M. S.; CARVALHO, F. Perfil demográfico e condições sanitárias dos idosos em área urbana do Sudeste do Brasil. Revista de Saúde Pública, São Paulo, v. 30, n. 5, p. 485-498, out. 1996.

Ciênc. agrotec., Lavras, v. 31, n. 2, p. 439-447, mar./abr., 2007 
TRUCKSESS, M. W.; MISLIVEC, P. B.; YOUNG, K.; BRUCE, V. R.; PAGE, S. W. Ciclopiazonic acid production by cultures of Aspergillus and Penicillium species isolated from dried beans, corn meal, macaroni and pecans. Journal of the Association Official Analytical Chemistry, Gainthersburg, v. 70, n. 1, p. 123-126, Jan./Feb. 1987.

URANO, T.; TRUCKSESS, M. W.; BEAVER, R. W.; WILSON, D. M.; DORNER, J. W.; DOWELL, F. E. Co-occurrence of cyclopiazonic acid and aflatoxins in corn and peanuts. Journal of the Association Official Analytical Chemistry, Gainthersburg, v. 75, n. 5, p. 838-841, Sept./Oct. 1992.
WOGAN, G. N.; EDQWARDS, G. S.; NEWBERNE, P. M. Structure-activity relationshipsin toxicity and carcinogenicity of aflatoxins and analogs. Cancer Research, Baltimore, v. 31, p. 1936-1942, 1971.

XIAO, H.; MADHYASTHA, S.; MARQUARDT, R. R.; LI, S.; VODELA, J. K.; FROHLICH, A. A.; KEMPPAINEN, B. W. Toxicity of ochratoxin A, its opened lactone from and several of its analogs: structure-activity relatonships. Toxicology and Applied Pharmacology, San Diego, v. 137, p. 182-192, 1996. 